\title{
Magnetic Structuring of Electrodeposits
}

\author{
Peter Dunne, Lorenzo Mazza, and J. M. D. Coey \\ School of Physics and CRANN, Trinity College, Dublin 2, Ireland
}

(Received 15 April 2011; published 5 July 2011)

\begin{abstract}
Metal electrodeposition reflects the pattern of the magnetic field at the cathode surface created by a magnet array. For deposits from paramagnetic cations such as $\mathrm{Co}^{2+}$ or $\mathrm{Cu}^{2+}$, the effect is explained in terms of magnetic pressure which modifies the thickness of the diffusion layer, that governs their mass transport. An inverse effect allows deposits to be structured in complementary patterns when a strongly paramagnetic but nonelectroactive cation such as $\mathrm{Dy}^{3+}$ is present in the electrolyte, and is related to inhibition of convection of water liberated at the cathode, in the inhomogeneous magnetic field. The magnetic structuring depends on the susceptibility of the electroactive species relative to that of the nonelectroactive background.
\end{abstract}

PACS numbers: 82.47.Wx, 47.65.Cb, 82.45.Qr

Magnetic fields often have a surprising influence on physical and chemical processes occurring in electrochemical cells. The rate of electrodeposition of metals [1-3], deposit morphology [4,5], hydrogen production [6,7], corrosion [8,9], rest potential [10], alloy composition [11,12], and magnetic properties [13], are all sensitive to applied magnetic field. The physical explanation is frequently magnetohydrodynamic, involving the Lorentz force [14]

$$
\mathbf{F}_{L}=\mathbf{j} \times \mathbf{B},
$$

where $\mathbf{j}$ is the current density in the cell and $\mathbf{B}$ is the applied magnetic field.

Another force is operative when the field is nonuniform and induces a magnetization, $\mathbf{M}=\chi \mathbf{H}$, in the electrolyte. Here $\chi$ is the susceptibility, which is expressed as $\chi_{m} c$ where $\chi_{m}$ is the molar susceptibility and $c$ is the concentration of ions in the electrolyte in $\mathrm{mol} \mathrm{m}^{-3}$. Since $\chi \ll 1$ for the electrolytes used in electrochemistry, the difference between $\mathbf{B}$ and $\mu_{0} \mathbf{H}$ can be neglected. The Kelvin force then takes the form $\mathbf{F}_{K}=\mu_{0} M \nabla H$. Provided the cell current is also negligible as a source of $\mathbf{H}$, the field gradient force can be written as $\mathbf{F}_{\nabla H}=\mu_{0} \chi H \nabla H$ or

$$
\mathbf{F}_{\nabla B}=\left(1 / 2 \mu_{0}\right) \chi_{m} c \nabla B^{2} .
$$

This may exceed the Lorentz force when the electrolyte is paramagnetic [15]. The ratio of the magnitudes of the two forces is

$$
R=\chi \nabla H / j .
$$

The magnetic field gradient does not drag ions in solution into the vicinity of a magnet, because the energy of a single ion with spin $S$ at temperature $T$ in the field $B$, $g^{2} \mu_{B}^{2} S(S+1) B^{2} / 6 \mathrm{kT}$, is about 5 orders of magnitude less than $\mathrm{kT}$, the thermal energy driving diffusion. $\left(\mu_{B} / \mathrm{kT}=\right.$ $0.67 \mathrm{KT}^{-1}$, where $\mu_{B}$ is the Bohr magneton and $k$ is Boltzmann's constant). Taking the curl of $\mathbf{F}_{\nabla B}$ it is possible to distinguish two regions where the influence of the field gradient force is different [16],

$$
\nabla \times \mathbf{F}_{\nabla B}=\left(1 / 2 \mu_{0}\right) \chi_{m}\left(\nabla c \times \nabla B^{2}\right)
$$

In the bulk solution, where there is no concentration gradient, the force is conservative, meaning it cannot induce convection, but it can modify or bifurcate existing convective flows. However, close to an electrode interface where a concentration gradient exists, the force is able to induce local convective flows. There is growing interest in the influence of nonuniform magnetic fields on electrochemical reactions, ranging from confinement of organic species at disc microelectrodes [17], remapping of MHD flows [18], electrodeposit patterning by magnetized iron wires [19], and spatially correlated suppression of corrosion of iron wires [20]. Here we show how electrodeposits of both magnetic and nonmagnetic cations can be structured using nonuniform fields. The deposits of magnetic ions are governed by the magnitude of the field at the cathode surface, and on the difference $\Delta \chi$ between the susceptibility of the electrolyte with and without electroactive cations. The ratio $R$ is $\gg 1$ in our experiments, so the field gradient force dominates.

Electrodeposits were produced from acidified solutions of the divalent cations $\mathrm{Co}^{2+}\left(3 d^{7}\right), \mathrm{Cu}^{2+}\left(3 d^{9}\right)$ and $\mathrm{Zn}^{2+}$ $\left(3 d^{10}\right)$. For cobalt, the divalent ion, monovalent ion and metal atom all have an unfilled $d$ shell and are paramagnetic, whereas for zinc all three are diamagnetic. In the case of copper, only the divalent ion is paramagnetic. In this way, it is possible to distinguish magnetic effects occurring at the electrode surface from those arising in the diffusion layer, a region of thickness $\delta \sim 100 \mu \mathrm{m}$ where a concentration gradient of $\mathrm{M}^{2+}$ drives the ions towards the cathode, where they are reduced to metal. The steady-state current density at overpotentials normally used for electrodeposition is limited by mass transport in the diffusion layer to a value

$$
\mathbf{j}_{0}=2 F D \nabla c,
$$


where $\nabla c=c_{0} / \delta_{c}, c_{0}$ is the bulk molar concentration of divalent cations in the electrolyte, $D$ is the ion diffusion constant and $F=96485 \mathrm{C} \mathrm{mol}^{-1}$ is Faraday's constant.

The cathode in the cell shown in Fig. 1 is a downwardfacing foil, usually either $50 \mu \mathrm{m}$ thick silver, or $17 \mu \mathrm{m}$ thick copper, backed by an array of $\mathrm{Nd}_{2} \mathrm{Fe}_{14} \mathrm{~B}$ permanent magnets. Many different arrangements of cylindrical or bar-shaped magnets have been investigated, including regular square and hexagonal arrays of cylindrical magnets with diameter $2 \mathrm{~mm}$, height $2 \mathrm{~mm}$ and spacing $3 \mathrm{~mm}$. We focus on square or hexagonal arrays where the adjacent magnets is aligned parallel or antiparallel. The arrays each included 80 magnets, but only 25-30 were within the area of the cathode of diameter $19 \mathrm{~mm}$ which was exposed to the electrolyte, in order to minimize magnetic edge effects. Figure 2 shows the distribution of magnetic field $250 \mu \mathrm{m}$ above the electrode surface, as well as the calculated flow patterns driven by the Lorentz force (1). The parallel arrays lead to circumferential flow, whereas the antiparallel arrays produce unfrustrated alternating flows around each magnet.

The solutions used were (a) $1 \mathrm{MZnCl}_{2}+0.4 \mathrm{M} \mathrm{H}_{3} \mathrm{BO}_{3}$ (b) $2 \mathrm{MCoSO}_{4}+0.6 \mathrm{M} \mathrm{H}_{3} \mathrm{BO}_{3}$, (c) $1 \mathrm{MCuSO}_{4}+$ $1.2 \mathrm{M} \mathrm{H}_{2} \mathrm{SO}_{4}+0.25 \mathrm{mM} \mathrm{KCl}$ and (d) $0.1 \mathrm{MCuSO}_{4}+$ $1 \mathrm{MNaSO}_{4}+0.1 \mathrm{M}$ acetic acid $+0.1 \mathrm{M}$ sodium acetate. Metals were deposited potentiostatically; bath (a) at $-2.0 \mathrm{~V}$ for $10 \mathrm{~min}$, bath (b) at $-1.2 \mathrm{~V}$ for $20 \mathrm{~min}$, bath (c) at $-0.4 \mathrm{~V}$ for $20 \mathrm{~min}$ and bath (d) at $-0.85 \mathrm{~V}$ for $10 \mathrm{~min}$. The bulk magnetic susceptibilities of the four solutions are (a) $-10 \times 10^{-6}$, (b) $250 \times 10^{-6}$, (c) $7.3 \times 10^{-6}$, and (d) $-0.7 \times 10^{-6}$, respectively. Little evidence of magnetic structuring was observed for zinc electrodeposits from bath (a), but cobalt and both copper solutions produced dot arrays of cobalt or copper which developed with time; an example is illustrated in Fig. 3. No patterning was observed in the first 10-20 s. There was no qualitative difference between the copper dot arrays produced from baths (c) and (d) with positive or negative bulk susceptibility. The critical factor is therefore the paramagnetic susceptibility of the electroactive ions in the solution, not the bulk susceptibility of the electrolyte. Results for the parallel and antiparallel arrays look quite similar. The thickness profiles measured with a DEKTAK $6 \mathrm{M}$ for different arrays show copper thicknesses after $600 \mathrm{~s}$ which



FIG. 1 (color online). Electrochemical cell used for producing magnetically structured deposits. The cathode is an $\mathrm{Ag}$ or $\mathrm{Cu}$ foil, backed by an array of magnets which are outside the cell. vary by a factor of 3-10 from a point between the magnets where $B_{z}$ is close to zero to a point directly beneath the magnets where $B_{z}=300 \mathrm{mT}$. Reducing the field by increasing the separation between the magnet array and the cathode had little effect, for separations up to $350 \mu \mathrm{m}$.

Hydrogen generation was negligible for the copper baths, but for the cobalt, bath (b) hydrogen bubbles grew in the interstices of the magnet arrays, leaving bald patches between the magnets.

Next we conducted a series of experiments where the electroactive copper or zinc was mixed with a dysprosium salt which has a high paramagnetic susceptibility $\chi_{\text {mol }}=$ $550 \times 10^{-9} \mathrm{~m}^{3} \mathrm{~mol}^{-1}$, but is not electroactive at accessible deposition potentials. The two baths used were (e) $0.1 \mathrm{MCuSO}_{4}+0.3 \mathrm{H}_{3} \mathrm{BO}_{3}+1 \mathrm{MDy}\left(\mathrm{NO}_{3}\right)_{3}$ and (f) $0.1 \mathrm{MZnCl}_{2}+0.4 \mathrm{M} \mathrm{H}_{3} \mathrm{BO}_{3}+1 \mathrm{MDyCl}_{3}$. Results for a hexagonal array are shown in Fig. 4. We see the inverse of the effect in Fig. 2. The copper or zinc now deposits preferentially in regions far from the magnets where the field is small, and where a diffusion layer thickness of order $300 \mu \mathrm{m}$ is established by a convective process which leads to turnover in the electrolyte. The antidots below the magnet arrays are thinly plated.

All our observations can be explained in terms of the magnetic field acting in the diffusion layer of the electrolyte. As mentioned earlier, we may safely ignore any direct influence of the field gradient force on diffusion.
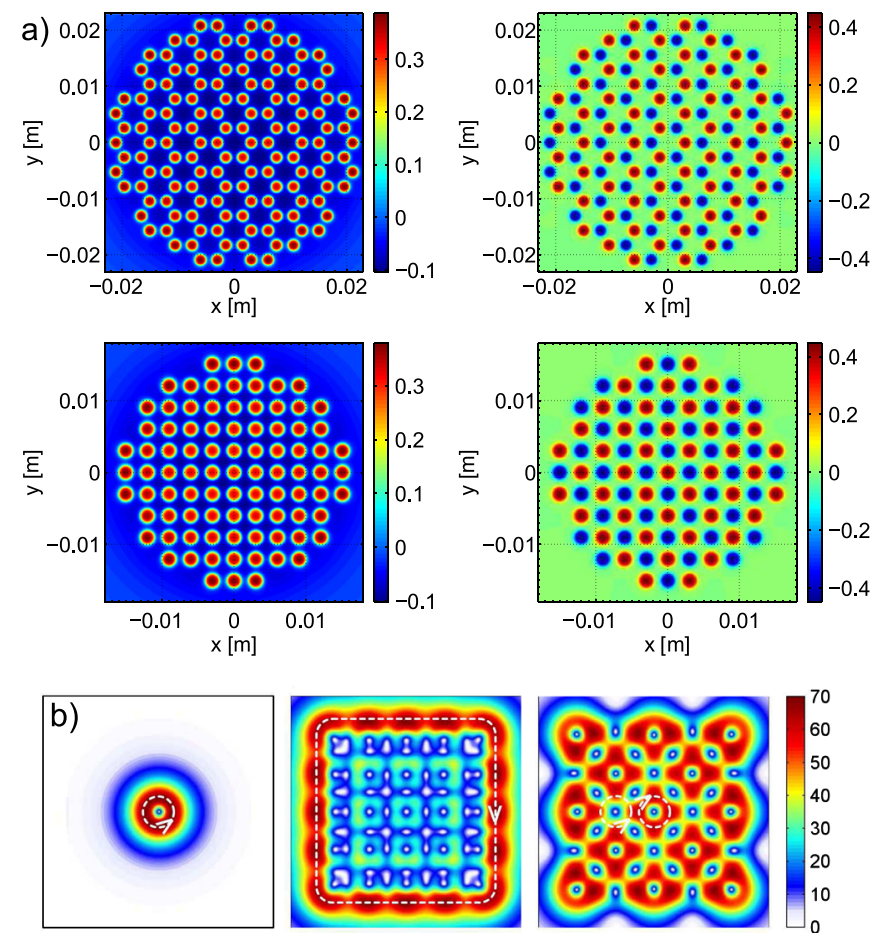

FIG. 2 (color online). Magnet arrays used for the magnetoelectrodeposition showing (a) contours of $B_{z}(T)$ for hexagonal parallel and antiparallel, and square parallel and antiparallel arrangements; (b) Flow patterns induced by the Lorentz force for a single magnet, a square parallel and square antiparallel arrays. 

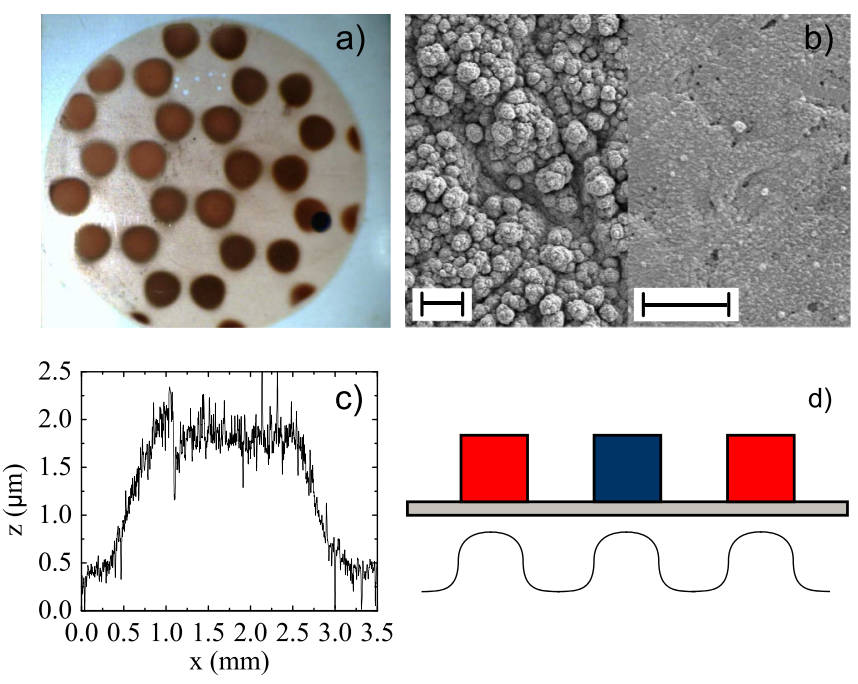

FIG. 3 (color online). (a) A silver cathode after electrodeposition from a $0.1 \mathrm{M}$ copper solution with an antiparallel hexagonal array (cf. Fig. 2); (b) SEM images of the copper surface above and between magnets, scale bars are $1 \mu \mathrm{m}$; (c) DEKTAK thickness profile of a copper dot; (d) scheme of the diffusion layer thickness.

However, the force (2) when $B=300 \mathrm{mT}, \quad \chi=$ $10 \times 10^{-6}$ and $\nabla B=200 \mathrm{~T} \mathrm{~m}^{-1}$ is of order $500 \mathrm{~N} \mathrm{~m}^{-3}$, which is comparable to the force density driving convection, $\Delta \rho \mathrm{g}$, where $\Delta \rho$ is the density difference between the ionic solution and pure water. The downward-facing cathode is intended to mitigate natural convection due to density differences in the electrolyte, but convective processes involving a backflow of water from the cathode are unavoidable and they stabilize the diffusion layer thickness after about $200 \mathrm{~s}$ in our cell. The hydration number of $\mathrm{Cu}^{2+}$ or $\mathrm{Zn}^{2+}$ is $\sim 18$ [21] so the volume of water shed is about 160 times the volume of metal deposited. It should also be noted that $R$ in Eq. ( 3$) \approx 50$ in these conditions.

The question is how can the deposition current be modified by the local magnetic field? The copper thicknesses in Fig. 3(c) indicate that $\nabla c$ varies by a factor 4 across the hexagonal magnet magnet array, as indicated schematically in Fig. 3(d). It follows from Eq. (5) that when $j=$ $31 \mathrm{~A} \mathrm{~m}^{-2}$ in a $0.1 \mathrm{M} \mathrm{Cu}^{2+}$ solution, for which the diffusion constant $D$ is $0.5 \times 10^{-9} \mathrm{~m}^{2} \mathrm{~s}^{-1}$, the average diffusion layer thickness is $\delta=311 \mu \mathrm{m}$.

To understand how the magnetic forces exerted on the electrolyte modulate the diffusion layer thickness, we consider the induced magnetization $\mathbf{M}$ of the electrolyte $M$. Forces are conveniently calculated using the magnetic charge model, which is a favorite approach for problems in magnetostatics. Wherever the magnetization is nonuniform, there is a distribution of bulk magnetic charge of

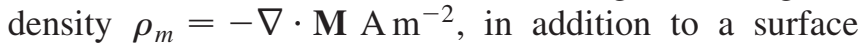
charge density at any sharp interface, $\sigma_{m}=M_{\perp} \mathrm{A} \mathrm{m}^{-1}$, where $M_{\perp}=\chi H_{\perp}$ is the component of magnetization perpendicular to the interface. The susceptibility $\chi$ is isotropic and very small, so demagnetizing effects can be neglected, and $\mathbf{M}=c \chi_{\mathrm{mol}} \mathbf{H}$ is everywhere parallel to the field produced by the magnet. In the region beyond the diffusion layer, the electrolyte concentration is uniform, and the corresponding distribution of magnetic charge is $\rho_{m}=-c_{0} \chi_{\mathrm{mol}} \nabla \cdot \mathbf{H}$. However, $\mathbf{B}=\mu_{0} \mathbf{H}$, since the contribution from $\mathbf{M}$ is neglected. But $\nabla \cdot \mathbf{B}=0$, so $\rho_{m}=0$. In other words, there is no magnetic force on any part of the electrolyte where the concentration is uniform. Furthermore, the cell is assumed to be deep enough to neglect the magnetization of the electrolyte at the lower cell wall. Since there is therefore no surface charge at the lower wall there is no force there either.

The force exerted by the magnet on the electrolyte can be therefore be calculated by considering only the diffusion layer, where $c<c_{0}$. The force on an induced magnetic charge $q_{m}$ is $(1 / 2) \mu_{0} H q_{m}$. The volume charge density is $-d M_{z} / d z$, so the force per unit area is

$$
P_{m}=\int_{0}^{\delta_{c}} \mu_{0} H_{z}\left(d M_{z} / d z\right) d z \approx \frac{1}{2} \mu_{0} \chi_{\mathrm{mol}} c_{0} H_{z}^{2} .
$$

When the variation of the magnetic field over the diffusion layer can be neglected, the magnetic pressure is equivalent to that on a sheet of surface charge $\sigma_{m}=M_{\perp}$ at $z=\delta$ where $\chi_{\mathrm{mol}}$ and $M$ refer to the susceptibility and magnetization associated with paramagnetic ions. There is no magnetic pressure on the diamagnetic water that fills the cell, because it has no free surface [22]. When $H_{z}=$ $240 \mathrm{kA} \mathrm{m}^{-2}$, Eq. (6) gives the upward magnetic pressure on the free layer for $0.1 \mathrm{M} \mathrm{CuSO}_{4}, c_{0}=100 \mathrm{~mol} \mathrm{~m}^{-3}$,

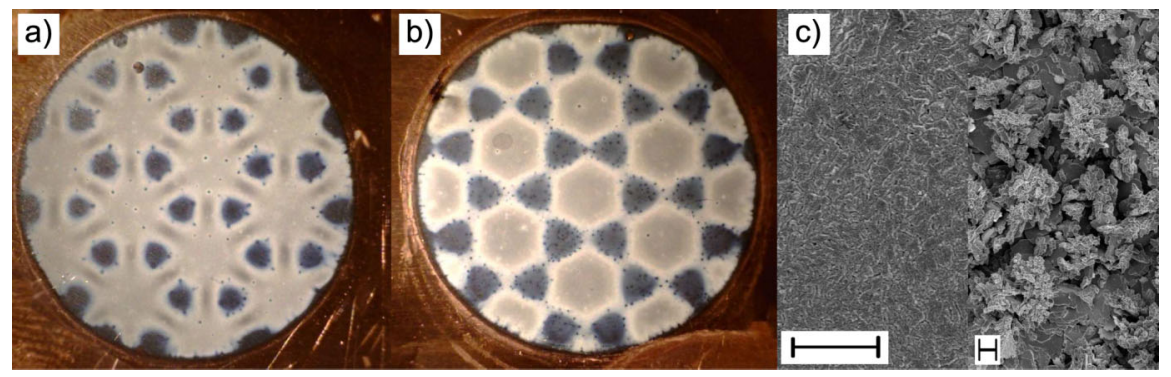

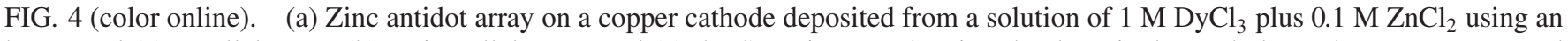
hexagonal (a) parallel array, (b) antiparallel array, and (c) the SEM images showing the deposited morphology above a magnet, and away from a magnet, scale bars are $2 \mu \mathrm{m}$. 
$\chi_{\text {mol }}=16 \times 10^{-9}$ as $57.6 \mathrm{mPa}$. The magnetic pressure balances the excess pressure due to the paramagnetic electrolyte, $P_{m}=(\delta(0)-\delta(H)) \Delta \rho g$. For $0.1 \mathrm{M} \mathrm{CuSO}$, $\Delta \rho=15 \mathrm{~kg} \mathrm{~m}^{-3}$ so the decrease in diffusion layer thickness below a magnet is $392 \mu \mathrm{m}$.

The ratio of 4 in the thickness in the copper profile across the dots in Fig. 3(c) $(\sim 0.45 \mu \mathrm{m}$ to $\sim 1.80 \mu \mathrm{m})$ is accounted for if the diffusion layer thickness is modulated by the magnetic field from 155 to $618 \mu \mathrm{m}$. (The dots occupy $1 / 3$ of the area of the deposit). The decrease in diffusion layer thickness below the magnet is $463 \mu \mathrm{m}$ compared to the estimate of $392 \mu \mathrm{m}$ from the magnetic pressure. While the agreement is well within experimental error in the thickness determinations, the analysis has neglected lateral diffusion as well as the horizontal components of magnetic field, which will deform the diffusion layer profile, leading to more angular dot shapes.

A somewhat different explanation is needed for the reverse patterning of Fig. 4. The magnetic pressure on $\mathrm{Zn}$ is small, because of its weak diamagnetic susceptibility. Moreover, the dysprosium-rich solution should be initially impervious to magnetic pressure for the same reason as water was; it is not depleted because the dysprosium is not reduced at the overpotentials used in the experiment $(<-2 \mathrm{~V})$ and there is no free surface, at least at the start of the experiment. However, the early stage of $\mathrm{Zn}$ deposition sheds water at the cathode surface, which creates a Dy interface which is subject to magnetic pressure. As the $\mathrm{Zn}$ diffusion layer is established, water and electroactive zinc solution are squeezed into the interstitial spaces between the magnets where the field is low. The difference for parallel and antiparallel arrays (Fig. 4) reflects the magnitude of the field at the cathode's surface.. Diffusive demixing of zinc and dysprosium ions cannot be driven by the magnetic field gradient, because the force (2), is too weak to influence diffusion, but a free surface can be stabilized in an inhomogeneous field. Once the electrolyte below the magnets is depleted of zinc after the initial stage of deposition, the convective process in the electrolyte column which would normally establish dynamic equilibrium is inhibited by the field gradient force, which establishes the segregation of zinc-rich regions between the magnets and zinc poor regions below them. The process resembles the stabilization of segregated water antitubes in a paramagnetic solution by a strong magnetic field gradient $\nabla B^{2}$ that we demonstrated earlier [23].

Although we have provided an account of the results in terms of mass-transport limited electrodeposition, there are significant magnetic field effects on the nucleation process, which are most important at low overpotential in the kinetically-controlled region. We will discuss nucleation and other short-time effects elsewhere.

In conclusion, we have shown how magnetic fields can be used to structure electrodeposits of both paramagnetic and diamagnetic ions. For arrays deposited from paramagnetic ions, the critical factor is the magnitude of the normal component of the magnetic field at the cathode surface $B_{z}$.
Structured deposition of diamagnetic ions such as $\mathrm{Zn}^{2+}$ is achieved by enhancing the field gradient force with magnetic contrast, and it depends on the magnitude of the total field $|\mathbf{B}|$. While we have focused here on dot arrays, other shapes of electrodeposits can be produced which mirror the distribution of the component of magnetic field normal to the cathode surface.

The scale of the deposits available will be limited by the diffusion layer thickness, which varies inversely with the current density. We have been able to deposit structured arrays of dots $80 \mu \mathrm{m}$ is size, but it is unlikely that this method could be extended below $10 \mu \mathrm{m}$.

This work was supported by Science Foundation Ireland as part of the RFP and NISE projects, contract numbers 09/RFP/PHY2372 and 10/IN1/I3002. We thank Dr. Plamen Stamenov for much helpful criticism.

[1] G. Hinds et al., J. Phys. Chem. B 105, 9487 (2001).

[2] M. Uhlemann, A. Krause, J. P. Chopart, and A. Gebert, J. Electrochem. Soc. 152, C817 (2005).

[3] P. Fricoteaux, B. Jonvel, and J.-P. Chopart, J. Phys. Chem. B 107, 9459 (2003).

[4] T.Z. Fahidy, Prog. Surf. Sci. 68, 155 (2001).

[5] J. A. Koza et al., Electrochim. Acta 55, 819 (2010).

[6] M. Uhlemann, A. Krause, and A. Gebert, J. Electroanal. Chem. 577, 19189 (2005).

[7] D. Fernández, Z. Diao, P. Dunne, and J. Coey, Electrochim. Acta 55, 8664 (2010).

[8] Z.H. Gu, J. Chen, A. Olivier, and T. Fahidy, J. Electrochem. Soc. 140, 408 (1993).

[9] A. Bund and H.H. Kuehnlein, J. Phys. Chem. B 109, 19845 (2005).

[10] F. M. F. Rhen and J. M. D. Coey, J. Phys. Chem. C 111, 3412 (2007).

[11] I. Tabakovic, S. Riemer, M. Sun, V. A. Vas'ko, and M. T. Kief, J. Electrochem. Soc. 152, C851 (2005).

[12] S. Pané, E. Gómez, and E. Vallés, J. Electroanal. Chem. 615, 117 (2008).

[13] T. Osaka et al., J. Electrochem. Soc. 139, 1311 (1992).

[14] R. Aogaki, K. Fueki, and T. Mukaibo, Denki Kagaku oyobi Kogyo Butsuri Kagaku 44, 89 (1976).

[15] G. Hinds, J. M. D. Coey, and M. E. G. Lyons, Electrochem. Comm. 3, 215 (2001).

[16] G. Mutschke et al., Electrochim. Acta 55, 9060 (2010).

[17] M. D. Pullins, K. M. Grant, and H. S. White, J. Phys. Chem. B 105, 8989 (2001).

[18] N. Leventis and X. Gao, Anal. Chem. 73, 3981 (2001).

[19] K. Tschulik, J. A. Koza, M. Uhlemann, A. Gebert, and L. Schultz, Electrochem. Comm. 11, 2241 (2009).

[20] R. Sueptitz, K. Tschulik, M. Uhlemann, A. Gebert, and L. Schultz, Electrochim. Acta 55, 5200 (2010).

[21] C. Akilan, G. Hefter, N. Rohman, and R. Buchner, J. Phys. Chem. B 110, 14961 (2006).

[22] The "Moses effect" where a magnetic field gradient forms a depression in a bath of water, requiring a free surface.

[23] J. M. D. Coey, R. Aogaki, F. Byrne, and P. Stamenov, Proc. Natl. Acad. Sci. U.S.A. 106, 8811 (2009). 